\title{
Detection of Neutral MSSM Higgs Bosons at LEP-II and NLC
}

\author{
U. Cotti ${ }^{a, *}$, A. Gutiérrez-Rodríguez ${ }^{b}$, A. Rosado ${ }^{b}$, and O. A. Sampayo ${ }^{c}$ \\ (a) SISSA-ISAS, via Beirut 2-4, 34013 Trieste, Italy. \\ (b) Instituto de Física, Universidad Autónoma de Puebla, \\ Apartado Postal J-48, Col. San Manuel, Puebla, Pue. 72570, México. \\ (c) Departamento de Física, Universidad Nacional de Mar de Plata, \\ Funes 3350, (7600) Mar de Plata, Argentina.
}

(February 23, 1999)

\begin{abstract}
We study the possibility of detecting the neutral Higgs bosons predicted in the Minimal Supersymmetric Standard Model $\left(h^{0}, H^{0}, A^{0}\right)$, with the reactions $e^{+} e^{-} \rightarrow b \bar{b} h^{0}\left(H^{0}, A^{0}\right)$, using the helicity formalism. We analyze the region of parameter space $\left(m_{A^{0}}-\tan \beta\right)$ where $h^{0}\left(H^{0}, A^{0}\right)$ could be detected in the limit when $\tan \beta$ is large. The numerical computation is done for the energy which is expected to be available at LEP-II $(\sqrt{s}=200 \mathrm{GeV})$ and for a possible Next Linear $e^{+} e^{-}$ Collider $(\sqrt{s}=500 \mathrm{GeV})$.
\end{abstract}

PACS: $14.80 . \mathrm{Cp}, 12.60 . \mathrm{Jv}$

\section{INTRODUCTION}

The Higgs sector is one of the most important areas of the Standard Model (SM) that has not been yet tested [1]. The SM contain only one neutral Higgs boson and although its detection would give more validity to the SM, there are some theoretical problems that suggest the need for new physics. One of the more attractive extensions of the SM is Supersymmetry (SUSY) [2], mainly because of its capacity to solve the naturalness and hierarchy problems while maintaining the Higgs bosons elementary.

The minimal supersymmetric extension of the Standard Model (MSSM) doubles the spectrum of particles of the SM and the new free parameters obey simple relations. The scalar sector of the MSSM [3] requires two Higgs doublets, thus the remaining scalar spectrum contains the following physical states: two CP-even Higgs scalar $\left(h^{0}\right.$ and $\left.H^{0}\right)$ with $m_{h^{0}} \leq m_{H^{0}}$, one CP-odd Higgs scalar $\left(A^{0}\right)$ and a charged Higgs pair $\left(H^{ \pm}\right)$, whose detection would be a clear signal of new physics. The Higgs sector is specified at tree-level by fixing two parameters, which can be chosen as the mass of the pseudoscalar $m_{A^{0}}$ and the ratio of vacuum expectation values of the two doublets $\tan \beta=v_{2} / v_{1}$, then the mass $m_{h^{0}}, m_{H^{0}}$ and $m_{H^{ \pm}}$and the mixing angle of the neutral Higgs sector $\alpha$ can be fixed. However, since radiative corrections produce substantial effects on the predictions of the model [4], it is necessary to specify also the squark masses, which are assumed to be degenerated. In this paper, we focus on the phenomenology of the neutral CP-even and CP-odd scalar $\left(h^{0}, H^{0}, A^{0}\right)$.

The search for these scalars has begun at LEP, and current low energy bound on their masses gives $m_{h^{0}}, m_{A^{0}}>$ $75 \mathrm{GeV}$ for $\tan \beta>1[5]$.

At $e^{+} e^{-}$colliders the signals for Higgs bosons are relatively clean and the opportunities for discovery and detailed study will be excellent. The most important processes for the production and detection of the neutral Higgs bosons, $h^{0}$, $H^{0}$ and $A^{0}$, are: $e^{+} e^{-} \rightarrow Z^{*} \rightarrow h^{0}, H^{0}+Z^{0}, e^{+} e^{-} \rightarrow Z^{*} \rightarrow h^{0}, H^{0}+A^{0}$, and $e^{+} e^{-} \rightarrow \nu \bar{\nu}+W^{+*} W^{-*} \rightarrow \nu \bar{\nu}+h^{0}, H^{0}$ (the later is conventionally referred to as $W W$ fusions); precise cross section formulae appear in Ref. [6]. There is considerable complementarity among the first four processes, and the $W W$ fusion processes are also complementary to one another and to the first four. If $m_{A^{0}} \gtrsim m_{Z^{0}}$, so that $\cos ^{2}(\beta-\alpha)$ is small, then $Z^{*} \rightarrow h^{0} Z^{0}, Z^{*} \rightarrow H^{0} A^{0}$, and $W W \rightarrow h^{0}$ are all maximal, and the other three small.

In particular, an $h^{0}$ with $m_{h^{0}} \sim m_{Z^{0}}$ could be seen at LEP-II provided that $\sqrt{s} \gtrsim 200 \mathrm{GeV}$ with $\mathcal{L} \sim 500 \mathrm{pb} b^{-}[7]$ can be achieved, and that efficient $b$-tagging is possible [8]. But, if the $h^{0}$ were not discovered at this energy, pushing slowly to $\sqrt{s}=240 \mathrm{GeV}$ would rapidly open up the possibility for $h^{0}$ detection in the regions of parameters space corresponding to the higher values of $M_{\tilde{t}}$ and $\tan \beta$. Of course, the ability of LEP-II to detect $h^{0}$ is also strongly dependent upon the actual $\sqrt{s}$ that can be achieved and upon the unknown value of $M_{\tilde{t}}$ (and to a lesser degree on the parameters that control squark mixing which in this paper will be neglected). Since radiative corrections make

*Permanent address: Centro de Investigación y de Estudios Avanzados del Instituto Politécnico Nacional, Apartado Postal 14-740, 07000 México, D.F. México 
possible to have $m_{h^{0}}>m_{Z^{0}}$, it seems that hadron colliders will be also required to test fully the Higgs sector of the MSSM [9].

The $Z^{0} h^{0}$ production cross section contains an overall factor $\sin ^{2}(\beta-\alpha)$ which suppress it in certain parameter regions (with $m_{A^{0}}<100 \mathrm{GeV}$ and $\tan \beta$ large); fortunately the $A^{0} h^{0}$ production cross section contains the complementary factor $\cos ^{2}(\beta-\alpha)$. Hence the $Z^{0} h^{0}$ and $A^{0} h^{0}$ channels together are well suited to cover all regions in the $\left(m_{A^{0}}-\tan \beta\right)$ plane, provided that the $c . m$. energy is high enough for $Z^{0} h^{0}$ to be produced through the whole $m_{h^{0}}$ mass range, and that an adequate event rate can be achieved. These conditions are already shown to be satisfied [10] for $\sqrt{s}=500 \mathrm{GeV}$ with assumed luminosity $10 \mathrm{fb}^{-1}$, like is expected to be the case of the Next Linear $e^{+} e^{-}$Collider (NLC).

In previous studies, the two body processes $e^{+} e^{-} \rightarrow h^{0}\left(H^{0}\right)+Z^{0}$ and $e^{+} e^{-} \rightarrow h^{0}\left(H^{0}\right)+A^{0}$ have been evaluated [6] extensively. However, the inclusion of three-body process $e^{+} e^{-} \rightarrow h^{0}\left(H^{0}\right)+b \bar{b}$ and $e^{+} e^{-} \rightarrow A^{0}+b \bar{b}$ at LEP-II and NLC energies is necessary in order to know its impact on the two body mode processes and also to search for new relations that could have a cleaner signature of the Higgs bosons production.

In the present paper we study the production of SUSY Higgs bosons at $e^{+} e^{-}$colliders. We are interested in finding regions that could allow the detection of the SUSY Higgs bosons for the set parameter space $\left(m_{A^{0}}-\tan \beta\right)$. We shall discuss the neutral Higgs bosons production $b \bar{b} h^{0}\left(H^{0}, A^{0}\right)$ in the energy range of LEP-II and NLC for large values of the parameter $\tan \beta$, where one expects to have a high production. Since the coupling $h^{0} b \bar{b}$ is proportional to $\sin \alpha / \cos \beta$, the cross section will receive a large enhancement factor when $\tan \beta$ is large. Similar situation occurs for $H^{0}$, whose coupling with $b \bar{b}$ is proportional to $\cos \alpha / \cos \beta$. The coupling of $A^{0}$ with $b \bar{b}$ is directly proportional to $\tan \beta$, thus the amplitude will always grow with $\tan \beta$. We consider the complete set of Feynman diagrams at tree-level and use the helicity formalism [11-17] for the evaluation of the amplitudes. The results obtained for the 3 -body processes are compared with the dominant mode 2 -body reactions for the plane $\left(m_{A^{0}}-\tan \beta\right)$. Succinctly, our aim in this work is to analyze how much the results of the Bjorken Mechanism (Fig. 1.4) would be enhanced by the contribution from the diagrams depicted in Figs. 1.1, 1.2, 1.3, 1.5 and 1.6, in which the SUSY Higgs boson is radiated by a $b(\bar{b})$ quark.

Recently, it has been shown that for large values of $\tan \beta$ detection of SUSY Higgs bosons is possible at FNAL and LHC [18]. In the papers cited in Ref.[18] the authors calculated the corresponding 3-body diagrams for hadron collisions. They pointed out the importance of a large bottom Yukawa coupling at hadron colliders and showed that the Tevatron collider may be a good place for detecting SUSY Higgs bosons. In the case of the hadron colliders the three body diagrams come from gluon fusion and this fact makes the contribution from these diagrams more important, due to the gluon abundance inside the hadrons. The advantage for the case of $e^{+} e^{-}$colliders is that the signals of the processes are cleaner.

This paper is organized as follows. We present in Sect. II the relevant details of the calculations. Sect. III contains the results for the process $e^{+} e^{-} \rightarrow b \bar{b} h^{0}\left(H^{0}, A^{0}\right)$ at LEP-II and NLC. Finally, Sect. IV contains our conclusions.

\section{HELICITY AMPLITUDE FOR NEUTRAL HIGGS BOSONS PRODUCTION}

When the number of Feynman diagrams is increased, the calculation of the amplitude is a rather unpleasant task. Some algebraic forms [19] can be used in it to avoid manual calculation, but sometimes the lengthy printed output from the computer is overwhelming, and one can hardly find the required results from it. The CALKUL collaboration [20] suggested the Helicity Amplitude Method (HAM) which can simplify the calculation remarkably and hence make the manual calculation realistic.

In this section we discuss the evaluation of the amplitudes at tree-level, for $e^{+} e^{-} \rightarrow b \bar{b} h^{0}\left(H^{0}, A^{0}\right)$ using the HAM [11-17]. This method is a powerfull technique for computing helicity amplitudes for multiparticle processes involving massles spin-1/2 and spin-1 particles. Generalization of this method that incorporates massive spin- $1 / 2$ and spin- 1 particles, are given in Ref. [17]. This algebra is easy to program and more efficient than computing the Dirac algebra.

A Higgs boson $h^{0}, H^{0}$ and $A^{0}$ can be produced in scattering $e^{+} e^{-}$via the following processes:

$$
\begin{aligned}
& e^{+} e^{-} \rightarrow b \bar{b} h^{0}, \\
& e^{+} e^{-} \rightarrow b \bar{b} H^{0}, \\
& e^{+} e^{-} \rightarrow b \bar{b} A^{0} .
\end{aligned}
$$

The diagrams of Feynman, which contribute at tree-level to the different reaction mechanisms are depicted in Figs. 1-2. Using the Feynman rules given by the Minimal Supersymmetric Standard Model (MSSM), as are summarized in Ref. [6], we can write the amplitudes for these reactions. For the evaluation of the amplitudes we have used the spinor-helicity technique of $\mathrm{Xu}$, Zhang and Chang [12] (denoted henceforth by XZC), which is a modification of the 
technique developed by the CALKUL collaboration [20]. Following XZC, we introduce a very useful notation for the calculation of the processes (1)-(3).

\section{A. Cases $b \bar{b} h^{0}$ and $b \bar{b} H^{0}$}

Let us consider the process

$$
e^{-}\left(p_{1}\right)+e^{+}\left(p_{2}\right) \rightarrow b\left(k_{2}\right)+\bar{b}\left(k_{3}\right)+h\left(k_{1}\right), \quad h=h^{0}, H^{0}
$$

in which the helicity amplitude is denoted by $\mathcal{M}\left(\lambda\left(e^{+}\right), \lambda\left(e^{-}\right), \lambda(b), \lambda(\bar{b})\right)$. The Feynman diagrams for this process are shown in Fig. 1. From this figure it follows that the amplitudes that correspond to each graph are:

$$
\begin{aligned}
& \mathcal{M}_{1}=-i C_{1} P_{Z^{0} b}\left(s_{13}\right) P_{Z^{0} e}(s) T_{Z^{\circ} b}^{\mu} T_{Z^{0} e \mu} \\
& \mathcal{M}_{2}=-i C_{2} P_{Z^{0} b}\left(s_{12}\right) P_{Z^{0} e}(s) T_{Z^{0} b}^{\mu} T_{Z^{0} e \mu} \\
& \mathcal{M}_{3}=-i C_{3} P_{A^{0} b}\left(s_{23}\right) P_{Z^{0} e}(s) T_{A^{0} b}^{\mu} T_{Z^{0} e \mu} \\
& \mathcal{M}_{4}=i C_{4} P_{Z^{0} b}\left(s_{23}\right) P_{Z^{0} e}(s) T_{Z^{0} b}^{\mu} T_{Z^{0} e \mu} \\
& \mathcal{M}_{5}=-i C_{5} P_{\gamma b}\left(s_{13}\right) P_{\gamma e}(s) T_{\gamma b}^{\mu} T_{\gamma e \mu} \\
& \mathcal{M}_{6}=-i C_{6} P_{\gamma b}\left(s_{12}\right) P_{\gamma e}(s) T_{\gamma b}^{\mu} T_{\gamma e \mu}
\end{aligned}
$$

where

$$
\begin{aligned}
C_{1} & = \begin{cases}g^{3} m_{b} \sin \alpha / 32 M_{W} \cos ^{2} \theta_{W} \cos \beta, & h=h^{0} \\
-g^{3} m_{b} \cos \alpha / 32 M_{W} \cos ^{2} \theta_{W} \cos \beta, & h=H^{0}\end{cases} \\
C_{2} & = \begin{cases}g^{3} m_{b} \sin \alpha / 32 M_{W} \cos ^{2} \theta_{W} \cos \beta, & h=h^{0} \\
-g^{3} m_{b} \cos \alpha / 32 M_{W} \cos ^{2} \theta_{W} \cos \beta, & h=H^{0}\end{cases} \\
C_{3} & = \begin{cases}g^{3} m_{b} \tan \beta \cos (\beta-\alpha) / 16 M_{W} \cos ^{2} \theta_{W}, & h=h^{0} \\
-g^{3} m_{b} \tan \beta \sin (\beta-\alpha) / 16 M_{W} \cos ^{2} \theta_{W}, & h=H^{0}\end{cases} \\
C_{4} & = \begin{cases}g^{3} M_{Z^{0}} \sin (\beta-\alpha) / 4 \cos ^{3} \theta_{W}, & h=h^{0} \\
g^{3} M_{Z^{0}} \cos (\beta-\alpha) / 4 \cos ^{3} \theta_{W}, & h=H^{0}\end{cases} \\
C_{5} & = \begin{cases}g m_{b} e^{2} Q_{e} Q_{b} \sin \alpha / 2 M_{W} \cos \beta, & h=h^{0} \\
-g m_{b} e^{2} Q_{e} Q_{b} \cos \alpha / 2 M_{W} \cos \beta, & h=H^{0}\end{cases} \\
C_{6} & = \begin{cases}g m_{b} e^{2} Q_{e} Q_{b} \sin \alpha / 2 M_{W} \cos \beta, & h=h^{0} \\
-g m_{b} e^{2} Q_{e} Q_{b} \cos \alpha / 2 M_{W} \cos \beta, & h=H^{0}\end{cases}
\end{aligned}
$$

while the propagators are

$$
\begin{aligned}
P_{Z^{0} b}\left(s_{13}\right) & =\frac{1}{\left(k_{1}+k_{3}\right)^{2}}, \\
P_{Z^{0}}(s) & =\frac{1}{\left(s-M_{Z^{0}}^{2}-i M_{Z^{0}} \Gamma_{Z^{0}}\right)}, \\
P_{Z^{0} b}\left(s_{12}\right) & =\frac{1}{\left(k_{1}+k_{2}\right)^{2}}, \\
P_{h b}\left(s_{23}\right) & =\frac{1}{\left[\left(k_{2}+k_{3}\right)^{2}-M_{h}^{2}-i M_{h} \Gamma_{h}\right]}, h=h^{0}, H^{0}, A^{0} \\
P_{Z^{0} b}\left(s_{23}\right) & =\frac{1}{\left[\left(k_{2}+k_{3}\right)^{2}-M_{Z^{0}}^{2}-i M_{Z^{0}} \Gamma_{Z^{0}}\right]}, \\
P_{\gamma b}\left(s_{13}\right) & =P_{Z^{0} b}\left(s_{13}\right), \\
P_{\gamma e}(s) & =\frac{1}{s}, \\
P_{\gamma b}\left(s_{12}\right) & =P_{Z^{0} b}\left(s_{12}\right),
\end{aligned}
$$

and the corresponding tensors 


$$
\begin{aligned}
T_{Z^{0} b}^{\mu} & =\bar{u}\left(k_{2}\right) \gamma^{\mu}\left(a_{b}-b_{b} \gamma_{5}\right)\left(\not k_{1}+k_{3}\right) v\left(\not k_{3}\right), \\
T_{Z^{0} e^{\mu}} & =\bar{v}\left(p_{2}\right) \gamma_{\mu}\left(a_{e}-b_{e} \gamma_{5}\right) u\left(p_{1}\right), \\
T_{Z^{0} b}^{\mu} & =\bar{u}\left(k_{2}\right)\left(\not k_{1}+\not k_{2}\right) \gamma^{\mu}\left(a_{b}-b_{b} \gamma_{5}\right) v\left(k_{3}\right), \\
T_{A^{0} b}^{\mu} & =\bar{u}\left(k_{2}\right) \gamma_{5}\left(k_{2}+k_{3}-k_{1}\right)^{\mu} v\left(k_{3}\right), \\
T_{Z^{0} b}^{\mu} & =\bar{u}\left(k_{2}\right) \gamma^{\mu}\left(a_{b}-b_{b} \gamma_{5}\right) v k_{3}, \\
T_{\gamma b}^{\mu} & =\bar{u}\left(k_{2}\right) \gamma^{\mu}\left(\not k_{1}+\not k_{3}\right) v\left(k_{3}\right), \\
T_{\gamma b \mu} & =\bar{v}\left(p_{2}\right) \gamma_{\mu} u\left(p_{1}\right), \\
T_{\gamma b}^{\mu} & =\bar{u}\left(k_{2}\right)\left(\not k_{1}+\not k_{2}\right) \gamma^{\mu} v\left(k_{3}\right) .
\end{aligned}
$$

In fact, we rearrange the tensors $T^{\prime}$ s in such a way that they become appropriate to a computer program. Then, following the rules from helicity calculus formalism [11-17] and using identities of the type

$$
\left\{\bar{u}_{\lambda}\left(p_{1}\right) \gamma^{\mu} u_{\lambda}\left(p_{2}\right)\right\} \gamma_{\mu}=2 u_{\lambda}\left(p_{2}\right) \bar{u}_{\lambda}\left(p_{1}\right)+2 u_{-\lambda}\left(p_{1}\right) \bar{u}_{-\lambda}\left(p_{2}\right),
$$

which is in fact the so called Chisholm identity, and

$$
\not p=u_{\lambda}(p) \bar{u}_{\lambda}(p)+u_{-\lambda}(p) \bar{u}_{-\lambda}(p),
$$

defined as a sum of the two projections $u_{\lambda}(p) \bar{u}_{\lambda}(p)$ and $u_{-\lambda}(p) \bar{u}_{-\lambda}(p)$.

The spinor products are given by

$$
\begin{aligned}
& s\left(p_{i}, p_{j}\right) \equiv \bar{u}_{+}\left(p_{i}\right) u_{-}\left(p_{j}\right)=-s\left(p_{j}, p_{i}\right), \\
& t\left(p_{i}, p_{j}\right) \equiv \bar{u}_{-}\left(p_{i}\right) u_{+}\left(p_{j}\right)=\left[s\left(p_{j}, p_{i}\right)\right]^{*} .
\end{aligned}
$$

Using Eqs. (9), (10) and (11), which are proved in Ref. [17], we can reduce many amplitudes to expressions involving only spinor products.

Evaluating the tensor products of (5) for each combination of $\left(\lambda, \lambda^{\prime}\right)$ with $\lambda, \lambda^{\prime}= \pm 1$ one obtains the following expressions

$$
\begin{aligned}
& \mathcal{M}_{1}(+,+)=F_{1} f_{1}^{+,+} s\left(k_{2}, p_{2}\right) t\left(p_{1}, k_{1}\right) s\left(k_{1}, k_{3}\right), \\
& \mathcal{M}_{1}(+,-)=F_{1} f_{1}^{+,-} s\left(k_{2}, p_{1}\right) t\left(p_{2}, k_{1}\right) s\left(k_{1}, k_{3}\right), \\
& \mathcal{M}_{1}(-,+)=F_{1} f_{1}^{-,+} t\left(k_{2}, p_{1}\right) s\left(p_{2}, k_{1}\right) t\left(k_{1}, k_{3}\right), \\
& \mathcal{M}_{1}(-,-)=F_{1} f_{1}^{-,-} t\left(k_{2}, p_{2}\right) s\left(p_{1}, k_{1}\right) t\left(k_{1}, k_{3}\right), \\
& \mathcal{M}_{2}(+,+)=F_{2} f_{2}^{+,+} t\left(k_{2}, k_{1}\right) s\left(k_{1}, p_{2}\right) t\left(p_{1}, k_{3}\right), \\
& \mathcal{M}_{2}(+,-)=F_{2} f_{2}^{+,-} t\left(k_{2}, k_{1}\right) s\left(k_{1}, p_{1}\right) t\left(p_{2}, k_{3}\right), \\
& \mathcal{M}_{2}(-,+)=F_{2} f_{2}^{-,+} s\left(k_{2}, k_{1}\right) t\left(k_{1}, p_{1}\right) s\left(p_{2}, k_{3}\right), \\
& \mathcal{M}_{2}(-,-)=F_{2} f_{2}^{-,-} s\left(k_{2}, k_{1}\right) t\left(k_{1}, p_{2}\right) s\left(p_{1}, k_{3}\right),
\end{aligned}
$$




$$
\begin{aligned}
& \mathcal{M}_{5}(+,+)=F_{5} s\left(k_{2}, k_{1}\right) t\left(k_{1}, p_{1}\right) s\left(p_{2}, k_{3}\right), \\
& \mathcal{M}_{5}(+,-)=F_{5} s\left(k_{2}, k_{1}\right) t\left(k_{1}, p_{2}\right) s\left(p_{1}, k_{3}\right), \\
& \mathcal{M}_{5}(-,+)=F_{5} t\left(k_{2}, k_{1}\right) s\left(k_{1}, p_{2}\right) t\left(p_{1}, k_{3}\right), \\
& \mathcal{M}_{5}(-,-)=F_{5} t\left(k_{2}, k_{1}\right) s\left(k_{1}, p_{1}\right) t\left(p_{2}, k_{3}\right), \\
& \mathcal{M}_{6}(+,+)=F_{6} s\left(k_{2}, p_{2}\right) t\left(p_{1}, k_{1}\right) s\left(k_{1}, k_{3}\right), \\
& \mathcal{M}_{6}(+,-)=F_{6} s\left(k_{2}, p_{1}\right) t\left(p_{2}, k_{1}\right) s\left(k_{1}, k_{3}\right), \\
& \mathcal{M}_{6}(-,+)=F_{6} t\left(k_{2}, p_{1}\right) s\left(p_{2}, k_{1}\right) t\left(k_{1}, k_{3}\right), \\
& \mathcal{M}_{6}(-,-)=F_{6} t\left(k_{2}, p_{2}\right) s\left(p_{1}, k_{1}\right) t\left(k_{1}, k_{3}\right),
\end{aligned}
$$

where

$$
\begin{aligned}
& F_{1}=-2 i C_{1} P_{Z^{0} b}\left(s_{13}\right) P_{Z^{0} e}(s), \\
& F_{2}=-2 i C_{2} P_{Z^{0} b}\left(s_{12}\right) P_{Z^{0} e}(s) \text {, } \\
& F_{3}=-i C_{3} P_{h b}\left(s_{23}\right) P_{Z^{0} e}(s) \text {, } \\
& F_{4}=2 i C_{4} P_{Z^{0} b}\left(s_{23}\right) P_{Z^{0} e}(s) \text {, } \\
& F_{5}=-2 i C_{5} P_{\gamma b}\left(s_{13}\right) P_{\gamma e}(s) \text {, } \\
& F_{6}=-2 i C_{6} P_{\gamma b}\left(s_{12}\right) P_{\gamma e}(s) \text {, }
\end{aligned}
$$

and

$$
\begin{aligned}
& f_{1}^{+,+}=f_{2}^{+,+}=f_{4}^{+,+}=\left(a_{b}-b_{b}\right)\left(a_{e}-b_{e}\right), \\
& f_{1}^{+,-}=f_{2}^{+,-}=f_{4}^{+,-}=\left(a_{b}-b_{b}\right)\left(a_{e}+b_{e}\right), \\
& f_{1}^{-,+}=f_{2}^{-,+,}=f_{4}^{-,+}=\left(a_{b}+b_{b}\right)\left(a_{e}-b_{e}\right), \\
& f_{1}^{-,-}=f_{2}^{-,-}=f_{4}^{-,-}=\left(a_{b}+b_{b}\right)\left(a_{e}+b_{e}\right), \\
& f_{3}^{+,+}=f_{3}^{-,+,}=\left(a_{e}-b_{e}\right), \\
& f_{3}^{+,-}=f_{3}^{-,-}=\left(a_{e}+b_{e}\right) .
\end{aligned}
$$

Here, $a_{e}=-1+4 \sin ^{2} \theta_{W}, b_{e}=1, a_{b}=-1+\frac{4}{3} \sin ^{2} \theta_{W}$ and $b_{b}=-1$, according to the experimental data [22].

\section{B. Case $b \bar{b} A^{0}$}

The Feynman diagrams that contribute to the process $e^{+} e^{-} \rightarrow b \bar{b} A^{0}$ to tree-level, are shown in Fig. 2. The corresponding amplitudes to each graph are:

$$
\begin{aligned}
& \mathcal{M}_{1}=C_{1} P_{Z^{0} b}\left(s_{13}\right) P_{Z^{0} e}(s) T_{Z^{0} b}^{\mu} T_{Z^{0} e \mu}, \\
& \mathcal{M}_{2}=C_{2} P_{Z^{0} b}\left(s_{12}\right) P_{Z^{0} e}(s) T_{Z^{0} b}^{\mu} T_{Z^{0} e \mu}, \\
& \mathcal{M}_{3}=C_{3} P_{Z^{0} H}\left(s_{23}\right) P_{Z^{0} e}(s) T_{Z^{0} H}^{\mu} T_{Z^{0} e \mu}, \\
& \mathcal{M}_{4}=C_{4} P_{Z^{0} h}\left(s_{23}\right) P_{Z^{0} e}(s) T_{Z^{0} h}^{\mu} T_{Z^{0} e \mu}, \\
& \mathcal{M}_{5}=C_{5} P_{\gamma b}\left(s_{13}\right) P_{\gamma e}(s) T_{\gamma b}^{\mu} T_{\gamma e \mu}, \\
& \mathcal{M}_{6}=C_{6} P_{\gamma b}\left(s_{12}\right) P_{\gamma e}(s) T_{\gamma b}^{\mu} T_{\gamma e \mu},
\end{aligned}
$$

where

$$
\begin{aligned}
& C_{1}=g^{3} m_{b} \tan \beta / 32 M_{W} \cos ^{2} \theta_{W}, \\
& C_{2}=C_{1}, \\
& C_{3}=-g^{3} m_{b} \cos \alpha \sin (\beta-\alpha) / 16 M_{W} \cos \beta \cos ^{2} \theta_{W}, \\
& C_{4}=-g^{3} m_{b} \sin \alpha \cos (\beta-\alpha) / 16 M_{W} \cos \beta \cos ^{2} \theta_{W}, \\
& C_{5}=g m_{b} e^{2} Q_{e} Q_{b} \tan \beta / 2 M_{W}, \\
& C_{6}=C_{5},
\end{aligned}
$$


The propagators are given in Eq. (7), while the tensors $T_{Z^{0} b}^{\mu}$ and $T_{Z^{0} e \mu}$ Eq. (8), and the new ones as follows.

$$
\begin{aligned}
& T_{Z^{0} b}^{\mu}=\bar{u}\left(k_{2}\right) \gamma_{5}\left(\not k_{1}+\not k_{2}\right) \gamma^{\mu}\left(a_{b}-b_{b} \gamma_{5}\right) v\left(k_{3}\right), \\
& T_{Z^{0} h}^{\mu}=\bar{u}\left(k_{2}\right)\left(k_{1}-k_{2}-k_{3}\right)^{\mu} v\left(k_{3}\right) .
\end{aligned}
$$

Making use of Eqs. (9)-(11), we can reduce the amplitude $\mathcal{M}$ to expressions that contain only spinor products. Finally, evaluating the tensor products in Eq. (20) for each combination of $\lambda, \lambda^{\prime}= \pm 1$ we obtain:

$$
\begin{aligned}
& \mathcal{M}_{1}(+,+)=2 G_{1} g_{1}^{+,+} t\left(k_{2}, k_{1}\right) s\left(k_{1}, p_{2}\right) t\left(p_{1}, k_{3}\right), \\
& \mathcal{M}_{1}(+,-)=2 G_{1} g_{1}^{+,-} t\left(k_{2}, k_{1}\right) s\left(k_{1}, p_{1}\right) t\left(p_{2}, k_{3}\right), \\
& \mathcal{M}_{1}(-,+)=2 G_{1} g_{1}^{-,+} s\left(k_{2}, k_{1}\right) t\left(k_{1}, p_{1}\right) s\left(p_{2}, k_{3}\right), \\
& \mathcal{M}_{1}(-,-)=2 G_{1} g_{1}^{-,-} s\left(k_{2}, k_{1}\right) t\left(k_{1}, p_{2}\right) s\left(p_{1}, k_{3}\right), \\
& \mathcal{M}_{2}(+,+)=2 G_{2} g_{2}^{+,+} t\left(k_{2}, p_{1}\right) s\left(p_{2}, k_{1}\right) t\left(k_{1}, k_{3}\right), \\
& \mathcal{M}_{2}(+,-)=2 G_{2} g_{2}^{+,-} t\left(k_{2}, p_{2}\right) s\left(p_{1}, k_{1}\right) t\left(k_{1}, k_{3}\right), \\
& \mathcal{M}_{2}(-,+)=2 G_{2} g_{2}^{-,+} s\left(k_{2}, p_{2}\right) t\left(p_{1}, k_{1}\right) s\left(k_{1}, k_{3}\right), \\
& \mathcal{M}_{2}(-,-)=2 G_{2} g_{2}^{-,-} s\left(k_{2}, p_{1}\right) t\left(p_{2}, k_{1}\right) s\left(k_{1}, k_{3}\right),
\end{aligned}
$$

$$
\begin{aligned}
& \mathcal{M}_{3}(+,+)=-G_{3} g_{3}^{+,+} s\left(k_{2}, k_{3}\right)\left[t\left(p_{2}, k_{2}\right) s\left(k_{2}, p_{1}\right)+t\left(p_{2}, k_{3}\right) s\left(k_{3}, p_{1}\right)-t\left(p_{2}, k_{1}\right) s\left(k_{1}, p_{1}\right)\right], \\
& \mathcal{M}_{3}(+,-)=-G_{3} g_{3}^{+,-} s\left(k_{2}, k_{3}\right)\left[s\left(p_{2}, k_{2}\right) t\left(k_{2}, p_{1}\right)+s\left(p_{2}, k_{3}\right) t\left(k_{3}, p_{1}\right)-s\left(p_{2}, k_{1}\right) t\left(k_{1}, p_{1}\right)\right], \\
& \mathcal{M}_{3}(-,+)=-G_{3} g_{3}^{-,+} t\left(k_{2}, k_{3}\right)\left[t\left(p_{2}, k_{2}\right) s\left(k_{2}, p_{1}\right)+t\left(p_{2}, k_{3}\right) s\left(k_{3}, p_{1}\right)-t\left(p_{2}, k_{1}\right) s\left(k_{1}, p_{1}\right)\right], \\
& \mathcal{M}_{3}(-,-)=-G_{3} g_{3}^{-,-} t\left(k_{2}, k_{3}\right)\left[s\left(p_{2}, k_{2}\right) t\left(k_{2}, p_{1}\right)+s\left(p_{2}, k_{3}\right) t\left(k_{3}, p_{1}\right)-s\left(p_{2}, k_{1}\right) t\left(k_{1}, p_{1}\right)\right], \\
& \mathcal{M}_{4}(+,+)=-G_{4} g_{4}^{+,+} t\left(k_{2}, k_{3}\right)\left[s\left(p_{2}, k_{2}\right) t\left(k_{2}, p_{1}\right)+s\left(p_{2}, k_{3}\right) t\left(k_{3}, p_{1}\right)-s\left(p_{2}, k_{1}\right) t\left(k_{1}, p_{1}\right)\right], \\
& \mathcal{M}_{4}(+,-)=-G_{4} g_{4}^{+,-} t\left(k_{2}, k_{3}\right)\left[t\left(p_{2}, k_{2}\right) s\left(k_{2}, p_{1}\right)+t\left(p_{2}, k_{3}\right) s\left(k_{3}, p_{1}\right)-t\left(p_{2}, k_{1}\right) s\left(k_{1}, p_{1}\right)\right], \\
& \mathcal{M}_{4}(-,+)=-G_{4} g_{4}^{-,+} s\left(k_{2}, k_{3}\right)\left[s\left(p_{2}, k_{2}\right) t\left(k_{2}, p_{1}\right)+s\left(p_{2}, k_{3}\right) t\left(k_{3}, p_{1}\right)-s\left(p_{2}, k_{1}\right) t\left(k_{1}, p_{1}\right)\right], \\
& \mathcal{M}_{4}(-,-)=-G_{4} g_{4}^{-,-} s\left(k_{2}, k_{3}\right)\left[t\left(p_{2}, k_{2}\right) s\left(k_{2}, p_{1}\right)+t\left(p_{2}, k_{3}\right) s\left(k_{3}, p_{1}\right)-t\left(p_{2}, k_{1}\right) s\left(k_{1}, p_{1}\right)\right],
\end{aligned}
$$

$$
\begin{aligned}
& \mathcal{M}_{5}(+,+)=-2 G_{5} t\left(k_{2}, p_{1}\right) s\left(p_{2}, k_{1}\right) t\left(k_{1}, k_{3}\right), \\
& \mathcal{M}_{5}(+,-)=-2 G_{5} t\left(k_{2}, p_{2}\right) s\left(p_{1}, k_{1}\right) t\left(k_{1}, k_{3}\right), \\
& \mathcal{M}_{5}(-,+)=2 G_{5} s\left(k_{2}, p_{2}\right) t\left(p_{1}, k_{1}\right) s\left(k_{1}, k_{3}\right), \\
& \mathcal{M}_{5}(-,-)=2 G_{5} s\left(k_{2}, p_{1}\right) t\left(p_{2}, k_{1}\right) s\left(k_{1}, k_{3}\right), \\
& \mathcal{M}_{6}(+,+)=2 G_{6} t\left(k_{2}, k_{1}\right) s\left(k_{1}, p_{2}\right) t\left(p_{1}, k_{3}\right), \\
& \mathcal{M}_{6}(+,-)=2 G_{6} t\left(k_{2}, k_{1}\right) s\left(k_{1}, p_{1}\right) t\left(p_{2}, k_{3}\right), \\
& \mathcal{M}_{6}(-,+)=-2 G_{6} s\left(k_{2}, k_{1}\right) t\left(k_{1}, p_{1}\right) s\left(p_{2}, k_{3}\right), \\
& \mathcal{M}_{6}(-,-)=-2 G_{6} s\left(k_{2}, k_{1}\right) t\left(k_{1}, p_{2}\right) s\left(p_{1}, k_{3}\right),
\end{aligned}
$$

where

$$
\begin{aligned}
& G_{1}=C_{1} P_{Z^{0} b}\left(s_{13}\right) P_{Z^{0} e}(s), \\
& G_{2}=C_{2} P_{Z^{0} b}\left(s_{12}\right) P_{Z^{0} e}(s), \\
& G_{3}=C_{3} P_{Z^{0} H}\left(s_{23}\right) P_{Z^{0} e}(s), \\
& G_{4}=C_{4} P_{Z^{0} h}\left(s_{23}\right) P_{Z^{0} e}(s), \\
& G_{5}=C_{5} P_{\gamma b}\left(s_{13}\right) P_{\gamma e}(s), \\
& G_{6}=C_{6} P_{\gamma b}\left(s_{12}\right) P_{\gamma e}(s),
\end{aligned}
$$

and 


$$
\begin{array}{ll}
g_{1}^{+,+}=g_{2}^{+,+}=f_{1}^{+,+}, & g_{3}^{+,+}=g_{4}^{+,+}=f_{3}^{+,+,}, \\
g_{1}^{+,-}=g_{2}^{+,-}=f_{1}^{+,-}, & g_{3}^{+,-}=g_{4}^{+,-}=f_{3}^{+,-}, \\
g_{1}^{-,+}=g_{2}^{-,+}=f_{1}^{-,+}, & g_{3}^{-,+}=g_{4}^{-,+}=f_{3}^{-,+}, \\
g_{1}^{-,-}=g_{2}^{-,-}=f_{1}^{-,-}, & g_{3}^{-,-}=g_{4}^{-,-}=f_{3}^{-,-} .
\end{array}
$$

The expressions for the $f_{1}^{+,+}, f_{1}^{+,-}, f_{1}^{-,+}, f_{1}^{-,-}, f_{3}^{+,+}, f_{3}^{+,-}, f_{3}^{-,+}$and $f_{3}^{-,-}$are given in Eq. (19).

After the evaluation of the amplitudes of the corresponding diagrams, we obtain the cross sections of the analyzed processes for each point of the phase space using Eqs. (12)-(17) and (23)-(28) by a computer program, which makes use of the subroutine RAMBO (Random Momenta Beautifully Organized). The advantages of this procedure in comparison to the traditional "trace technique" are discussed in Ref. [11-17].

We use the Breit-Wigner propagators for the $Z^{0}, h^{0}, H^{0}$ and $A^{0}$ bosons. The mass of the bottom $\left(m_{b} \approx 4.5 \mathrm{GeV}\right)$, the mass $\left(M_{Z^{0}}=91.2 \mathrm{GeV}\right)$ and width $\left(\Gamma_{Z^{0}}=2.4974 \mathrm{GeV}\right)$ of $Z^{0}$ have been taken as inputs; the widths of $h^{0}, H^{0}$ and $A^{0}$ are calculated from the formulae given in Ref. [6]. In the next sections we present the numerical computation of the processes $e^{+} e^{-} \rightarrow b \bar{b} h, h=h^{0}, H^{0}, A^{0}$.

\section{DETECTION OF NEUTRAL HIGGS BOSONS AT LEP-II AND NLC ENERGIES}

In an earlier paper [21] has been explored the possibility of finding one or more of the neutral Higgs bosons predicted by the MSSM in $g g \rightarrow b \bar{b} h\left(h=h^{0}, H^{0}, A^{0}\right)$ followed by $h \rightarrow b \bar{b}$, profiting from the very high $b$-tagging efficiencies. In other works [18], it has been studied the discovery reach of the Tevatron and the LHC for detecting a Higgs boson $(h)$ via the processes $p \bar{p} / p p \rightarrow b \bar{b} h(\rightarrow b \bar{b})+X$ and it has been shown the possibility of detecting SUSY Higgs bosons at FNAL and LHC if $\tan \beta$ is large.

In this paper, we study the detection of neutral MSSM Higgs bosons at $e^{+} e^{-}$colliders, including 3-body mode diagrams (Figs. 1.1, 1.2, 1.3, 1.5 and 1.6; Figs. 2.1, 2.2, 2.3, 2.5 and 2.6) besides the dominant mode diagram (Fig. 1.4; Fig. 2.4) assuming an integrated luminosity of $\mathcal{L}=500 \mathrm{pb}^{-1}$ and $\mathcal{L}=10 \mathrm{fb}^{-1}$ at $\sqrt{s}=200 \mathrm{GeV}$ and $500 \mathrm{GeV}$ for LEP-II and NLC, respectively. We consider the complete set of Feynman diagrams (Fig. 1-2) at tree-level and utilize the helicity formalism for the evaluation of their amplitudes. In the next subsections, we present our results for the case of the different Higgs bosons.

\section{A. Detection of $h^{0}$}

In order to illustrate our results on the detection of the $h^{0}$ Higgs boson, we present graphs in the parameters space region $\left(m_{A^{0}}-\tan \beta\right)$, assuming $m_{t}=175 \mathrm{GeV}, M_{\tilde{t}}=500 \mathrm{GeV}$ and $\tan \beta>1$ for LEP-II and NLC. Our results are displayed in Fig. 3, for $e^{+} e^{-} \rightarrow\left(A^{0}, Z^{0}\right)+h^{0}$ dominant mode and for the processes at 3-body $e^{+} e^{-} \rightarrow b \bar{b} h^{0}$.

The total cross section for each contour is $0.01 p b, 0.02 p b$ and $0.05 p b$, which gives 5 events, 10 events and 25 events, respectively. We can see from this figure, that the effect of the reaction $b \bar{b} h^{0}$ is lightly more important that $\left(A^{0}, Z^{0}\right)+h^{0}$, for most of the $\left(m_{A^{0}}-\tan \beta\right)$ parameter space regions. Nevertheless, there are substantial portions of parameters space in which the discovery of the $h^{0}$ is not possible using either $\left(A^{0}, Z^{0}\right)+h^{0}$ or $b \bar{b} h^{0}$.

For the case of NLC, the results on the detection of the $h^{0}$ are shown in Fig. 4. It is clear from this figure that the contribution of the process $e^{+} e^{-} \rightarrow b \bar{b} h^{0}$ becomes dominant, namely $e^{+} e^{-} \rightarrow\left(A^{0}, Z^{0}\right)+h^{0}$ is small in all parameter space. However, they could provide important information on the Higgs bosons detection. For instance, we give the contours for the total cross section to say $0.01 \mathrm{pb}$ and $0.03 \mathrm{pb}$ for both processes. These cross sections give 100 events and 300 events in total to each process, then it will be detectable the $h^{0}$ at NLC energies.

\section{B. Detection of $H^{0}$}

The detection of the heavy Higgs bosons $H^{0}$ is not possible at LEP-II. Nevertheless, the possibility for a future $e^{+} e^{-}$collider with center of mass energy of order $500 \mathrm{GeV}$ is surveyed. Consequently, detection of the $H^{0}$ and $A^{0}$ will be only possible in such a scenario if $\sqrt{s}$ is significantly larger than $m_{A^{0}}+m_{H^{0}}$, i.e. $\gtrsim 2 m_{A^{0}}$ for large $m_{A^{0}}$ where $m_{H^{0}} \approx m_{A^{0}}$. To illustrate more precisely our results, we give the contours for the total cross section for both processes $e^{+} e^{-} \rightarrow\left(A^{0}, Z^{0}\right)+H^{0}, e^{+} e^{-} \rightarrow b \bar{b} H^{0}$ in Fig. 5 for an NLC with $\sqrt{s}=500 \mathrm{GeV}$ and $\mathcal{L}=10 \mathrm{fb}^{-1}$ in the case where $m_{t}=175 \mathrm{GeV}$ and $M_{\tilde{t}}=500 \mathrm{GeV}$ (squark mixing is neglected). The contours for this cross section are $0.01 \mathrm{pb}$, 
$0.001 p b$ and $0.0001 p b$ for both reactions $\left(A^{0}, Z^{0}\right)+H^{0}$ and $b \bar{b} H^{0}$. While that the number of events corresponding to each contour are 100, 10 and 1 , respectively.

Our estimate is that if more than 100 total events are obtained for a given process $\left(A^{0}, Z^{0}\right)+H^{0}$ or $b \bar{b} H^{0}$ then the Higgs boson $H^{0}$ can be detectable. Contours for 10 events are also shown, but detection of any of the two cases with so few events would require very high experimental and analysis efficiencies.

The effect of incorporate $b \bar{b} H^{0}$ in the detection of the Higgs boson $H^{0}$ is more important than the case of 2-body mode $\left(A^{0}, Z^{0}\right)+H^{0}$, because $b \bar{b} H^{0}$ cover a major region in the parameters space $\left(m_{A^{0}}-\tan \beta\right)$. The most important conclusion from this figure is that detection of all of the neutral Higgs bosons will be possible at $\sqrt{s}=500 \mathrm{GeV}$.

\section{Detection of $A^{0}$}

For the pseudoscalar $A^{0}$, it is interesting to consider the production mode into $b \bar{b} A^{0}$, since it can have large cross section due to that the coupling of $A^{0}$ with $b \bar{b}$ is directly proportional to $\tan \beta$, thus will always grow with it. In Fig. 6 , we present the contours of the cross sections for the process of our interest $b \bar{b} A^{0}$, at LEP-II energies.

We display the contour lines for $\sigma=0.01,0.02$, showing also the regions where the $A^{0}$ can be detected. These cross sections give a contour of production of 5 and 10 events. It is clear from this figure that to detecting the Higgs boson $A^{0}$ are necessary very high experimental and analysis efficiencies.

On the other hand, if we focus the detection of the $A^{0}$ at Next Linear $e^{+} e^{-}$Collider with $\sqrt{s}=500 \mathrm{GeV}$ and $\mathcal{L}=10 \mathrm{fb}^{-1}$, the panorama for its detection is more extensive. The Fig. 7, show the contours lines in the plane $\left(m_{A^{0}}-\tan \beta\right)$, to the cross section of $b \bar{b} A^{0}$. The contours for this cross section correspond to 100,30 and 10 events. It is estimated that if more than 100 total events are obtained for $b \bar{b} A^{0}$, then it is possible to detect the $A^{0}$.

\section{CONCLUSIONS}

In this paper, we have calculated the production of a neutral Higgs bosons in association with $b$-quarks via the processes $e^{+} e^{-} \rightarrow b \bar{b} h, h=h^{0}, H^{0}, A^{0}$ and using the helicity formalism. We find that this processes could help to detect a possible neutral Higgs boson at LEP-II and NLC energies when $\tan \beta$ is large.

The detection of $h^{0}$ through of the reaction $e^{+} e^{-} \rightarrow b \bar{b} h^{0}$, compete favorable with the mode dominant $e^{+} e^{-} \rightarrow$ $\left(A^{0}, Z^{0}\right)+h^{0}$. The process at 3 -body $b \bar{b} h^{0}$ cover lightly a major portion of the parameter space $\left(m_{A^{0}}-\tan \beta\right)$ as is shown in Fig. 3 and the corresponding cross section for each contour is of $\sigma=0.01 p b, 0.02 p b, 0.05 p b$ for LEP-II; while for NLC we have that $\sigma=0.01 p b, 0.03 p b$ and the corresponding contours are show in Fig. 4. However, there is a portion of the plane $\left(m_{A^{0}}-\tan \beta\right)$ where $h^{0}$ is not detectable with the mechanism $\left(A^{0}, Z^{0}\right)+h^{0}$ or $b \bar{b} h^{0}$.

The heavy Higgs boson $H^{0}$ is not detectable at LEP-II energies. Nevertheless, it could be detected at NLC via the $\left(A^{0}, Z^{0}\right)+H^{0}$ or $b \bar{b} h^{0}$ reaction, with $H^{0}$ being produced in association with $b$-quarks. The detection of the $H^{0}$ will only be possible in such scenarios if $\sqrt{s}$ is significantly larger that $m_{A^{0}}+m_{H^{0}}$, i.e. $\gtrsim 2 m_{A^{0}}$ for large $m_{A^{0}}$ where $m_{H^{0}} \approx m_{A^{0}}$. In Fig. 5, we give the contours for the cross sections for both cases $\left(A^{0}, Z^{0}\right)+H^{0}$ and $b \bar{b} H^{0}$. We find that for $m_{A^{0}}$ and $\tan \beta$ large the reaction $e^{+} e^{-} \rightarrow b \bar{b} H^{0}$ is more important that $e^{+} e^{-} \rightarrow\left(A^{0}, Z^{0}\right)+H^{0}$ and cover a major region in the parameter space $\left(m_{A^{0}}-\tan \beta\right)$ where this mode is kinematically allowed.

It is interesting to notice that the associate production of $A^{0}$ with $b \bar{b}$ is enhanced for larger values of $\tan \beta$, which could be used to detect neutral Higgs bosons, provided that it will be possible also to tag the signals using the $b$-quarks produced in the reaction.

In the Figs. 6-7, we show the contours for the total cross section of the process $b \bar{b} A^{0}$ for both LEP-II and NLC energies. We can conclude that there is a region where the Higgs boson $A^{0}$ could be detected at the next high energy machines (NLC).

In summary, we conclude that the possibilities of detecting or excluding the neutral Higgs bosons of the Minimal Supersymmetric Standard Model $\left(h^{0}, H^{0}, A^{0}\right)$ in the processes $e^{+} e^{-} \rightarrow b \bar{b} h, h=h^{0}, H^{0}, A^{0}$ are important and in some cases are compared favorable with the mode dominant $e^{+} e^{-} \rightarrow\left(A^{0}, Z^{0}\right)+h, h=h^{0}, H^{0}, A^{0}$ in the region of parameters space $\left(m_{A^{0}}-\tan \beta\right)$ with $\tan \beta$ large. The detection of the Higgs boson will require the combined use of the future high energy machine like LEP-II and the Next Linear $e^{+} e^{-}$Collider.

\section{Acknowledgments}

This work was supported in part by Consejo Nacional de Ciencia y Tecnología (CONACyT) and Sistema Nacional de Investigadores (SNI) (México). O. A. S. would like to thank CONICET (Argentina). We would like to thank J. L. Díaz-Cruz for suggestions and for careful reading of our manuscript. 


\section{FIGURE CAPTIONS}

Fig. 1 Feynman Diagrams at tree-level for $e^{+} e^{-} \rightarrow b \bar{b} h^{0}$. For $e^{+} e^{-} \rightarrow b \bar{b} H^{0}$ one has to make only the change $\sin \alpha / \cos \beta \rightarrow \cos \alpha / \cos \beta$.

Fig. 2 Feynman Diagrams at tree-level for $e^{+} e^{-} \rightarrow b \bar{b} A^{0}$.

Fig. 3 Total cross sections contours in $\left(m_{A^{0}}-\tan \beta\right)$ parameter space for $e^{+} e^{-} \rightarrow\left(A^{0}, Z^{0}\right)+h^{0}$ and $e^{+} e^{-} \rightarrow b \bar{b} h^{0}$ at LEP-II with $\sqrt{s}=200 \mathrm{GeV}$ and an integrated luminosity of $\mathcal{L}=500 \mathrm{pb}^{-1}$. We have taken $m_{t}=175 \mathrm{GeV}$ and $M_{\widetilde{t}}=500 \mathrm{GeV}$ and neglected squark mixing.

Fig. 4 Total cross sections contours for an NLC with $\sqrt{s}=500 \mathrm{GeV}$ and $\mathcal{L}=10 \mathrm{fb}^{-1}$. We have taken $m_{t}=175$ $\mathrm{GeV}, M_{\tilde{t}}=500 \mathrm{GeV}$ and neglected squark mixing. We display contours for $e^{+} e^{-} \rightarrow\left(A^{0}, Z^{0}\right)+h^{0}$ and $e^{+} e^{-} \rightarrow b \bar{b} h^{0}$, in the parameters space $\left(m_{A^{0}}-\tan \beta\right)$.

Fig. 5 Same as in Fig. 4, but for $e^{+} e^{-} \rightarrow\left(A^{0}, Z^{0}\right)+H^{0}$ and $e^{+} e^{-} \rightarrow b \bar{b} H^{0}$.

Fig. 6 Same as in Fig. 3, but for $e^{+} e^{-} \rightarrow b \bar{b} A^{0}$.

Fig. 7 Same as in Fig. 4, but for $e^{+} e^{-} \rightarrow b \bar{b} A^{0}$. 
[1] S. Weinberg, Phys. Rev. Lett. 19, (1967) 1264; A. Salam, in Elementary Particle Theory, ed. N. Southolm (Almquist and Wiksell, Stockholm, 1968), p.367; S.L. Glashow, Nucl. Phys. 22, (1967) 257.

[2] H. P. Nilles, Phys. Rep. 110, (1984) 1; H. Haber and G. L. Kane, Phys. Rep. 117, (1985) 75.

[3] J. Gunion and H. Haber, Nucl. Phys. 272, (1986) 1.

[4] S.P. Li and M. Sher, phys. Lett. B149, (1984) 339; J. F. Gunion and A. Turski, Phys. Rev. D39, (1989) 2701; 40, (1989) 2325; 40, (1989) 2333; Y. Okada et al. Prog. Theor. Phys. Lett. 85, (1991) 1; H.Haber and R. Hempfling, Phys. Rev. Lett. 66, (1991) 1815; J. Ellis et al., Phys. Lett. B257, (1991) 83; M. Dress and M. N.Nojiri, Phys. Rev. D45, (1992) $2482 ;$ M. A. Diaz and H. E. Haber, Phys. Rev. D45, (1992) 4246; D. M. Pierce, A. Papadoupoulos and S. Jonhson, Phys. Rev. Lett. 68, (1992) 3678; P. H. Chankowski, S. Poporski and J. Rosiek, Phys. Lett. B274, (1992) 191; A. Yamada, Mod. Phys. Lett. A7, (1992) 2877.

[5] A. Sopczak, hep-ph/9712283, IEKP-KA/97-14, 1997.

[6] For a recent review see J. Gunion, H. Haber, G. Kane and S. Dowson, The Higgs Hunter's Guide (Addison-Wesley, Reading, MA,1990).

[7] C. Rubbia, in Proceedings of the Joint International Lepton-Photon Symposium and Europhysics Conference on High Energy Physics, Geneva Switzerland, 1991, edited by S. Hergarty, K. Potter, and E. Quercigh (World Scientific, Singapore, 1992), p. 439; V. Barger, Kingman Cheung, R. N. J. Phillips and A. L. Stange, Phys. Rev. D47, (1993) 3041. J. F. Gunion, L. Poggioli, R. Van Kooten, C. Kao, P. Rowson, Higgs Boson Discovery and Properties, hep-ph/9703330, UCD-97-5, March 1997.

[8] V. Barger and K. Whisnant, Phys. Rev. D43, (1991) 1443. J. F. Gunion, Detecting the Supersymmetric Higgs Bosons, Perspectives on Higgs Physics, Ed. Gordon L. Kane, World Scientific, Singapore 1993; André Sopczak, Invited review talk given at the European Meeting of the Working Groups on Physics and Experiments at Linear $e^{+} e^{-}$Colliders, LAPPAnnecy-le-Viex, February, 1993.

[9] Z. Kunst and Zwirner, Nucl. Phys. B385, (1992) 3; J. Gunion and L. Orr, Phys. Rev. D46, (1992) 2052; J. L. Díaz-Cruz, Nucl. Phys. B358, (1991), 97; J. L. Díaz-Cruz and O. A. Sampayo, Int. J. Mod. Phys. A8, (1993) 4339.

[10] P. Janot, Orsay Report No. LAL 91-61, 1991 (unpublished); J-F Grivas, Orsay Report No. LAL 91-63, 1991 (unpublished) A. Brignole, J. Ellis, J. F. Gunion, M. Guzzo, F. Olnes, G. Ridolfi, L. Roszkowski and F. Zwirner, in $e^{+} e^{-}$Collision at $500 \mathrm{GeV}$ : The Physics Potential, Munich, Annecy, Hamburg Workshop, DESY 92-123A, DESY 92-123B, DESY 93-123C, ed. P. Zerwas, p. 613; A. Djouadi, J. Kalinowski, P. M. Zerwas, ibid.; p.83 and Z. Phys. C57, (1993) 56; and references therein; Physics and Technology of the Next Linear: a Report Submited to Snowmass 1996, BNL 52-502, FNAL-PUB -96/112, LBNL-PUB-5425,SLAC Report 485, UCRL-JD-124160; J. F. Gunion, Detection and Studying Higgs Bosons, hep-ph/9705282, UCD-97-13, 1997.

[11] Howard E. Haber, Proceedings of the 21st SLAC Summer Institute on Particle Physics: Spin Structure in High Energy Process SLAC, Stanford, CA, 26 July-6 August 1993.

[12] Zhan XU, Da-Hua ZHANG and Lee CHANG, Nucl. Phys. B291, (1987) 392.

[13] J. Werle, Relativistic Theory of Reactions (North-Holland, Amsterdam, 19976); A. D. Martin and T. D. Spearman, Elementary Particle Physics (North-Holland, Amsterdam, 1970); S. U. Chung, Spin Formalism, CERN Yellow Report 71-8 (1971); Martin L. Perl, High Energy Hadron Physics (John Wiley Sons, New York, 1974).

[14] H. Pilkum, The Interactions of Hadrons (North-Holland, Amsterdam, 1967).

[15] Peter A. Carruthers, Spin and Isospin in Particle Physics (Gordon and Breach, New York, 1971); M. D. Scadron, Advanced Quantum Theory (Springer-Verlag, Berlin, 1991).

[16] M. L. Mangano and S. J. Parke, Phys. Rep. 200, (1991) 301.

[17] F. A. Berends, P. H. Daverveldt and R. Kleiss, Nucl. Phys. B253, (1985) 441; R. Kleiss and W. J. Stirling, Nucl. Phys. B262, (1985) 235; C. Mana and M. Martinez, Nucl. Phys. B278, (1987) 601.

[18] J. Lorenzo Díaz-Cruz, Hong-Jian He, Tim Tai, C.-P. Yuan, Phys. Rev. Lett. 80, (1998) 4641; M. Carena, S. Mrenna and C. E. M. Wagner, MSSM Higgs Boson Phenomenology at the Tevatron Collider, hep-ph/9808312v2; C. Balázs, J. Lorenzo Díaz-Cruz, Hong-Jian He, Tim Tai, C.-P. Yuan, Probing Higgs Bosons with Large Bottom Yukawa Coupling at Hadron Colliders, hep-ph/9807349.

[19] A. C. Hearn, REDUCE User's Manual, version 3.2 (Rand Publ. CP78 Rev. 4/85, Santa Monica, CA. 1985); H. Strubbe, Comput. Phys. Commun. 8, (1974) 1.

[20] P. De Causmaecker, R. Gastmans, W. Troost and T. T. Wu, Phys. Lett. B105, (1981) 215; P. De Causmaecker, R. Gastmans, W. Troost and T. T. Wu, Nucl. Phys. B206, (1982) 53; F. A. Berends, R. Kleiss, P. De Causmaecker, R. Gastmans, W. Troost and T. T. Wu, Nucl. Phys. B206, (1982) 61; D. Dankaert, P. De Causmaecker, R. Gastmans, W. Troots and T. T. Wu, Phys. Lett. B114, (1982) 203; F. A. Berends, P. De Causmaecker, R. Gastmans, R. Kleiss, W. Troost and T. T. Wu, Nucl. Phys. B239, (1984) 382; F. A. Berends, P. De Causmaecker, R. Gastmans, R. Kleiss, W. Troost and T. T. Wu, Nucl. Phys. B239, (1984) 395; F. A. Berends, P. De Causmaecker, R. Gastmans, R. Kleiss, W. 
Troost and T. T. Wu, Nucl. Phys. B264, (1986) 234; F. A. Berends, P. De Causmaecker, R. Gastmans, R. Kleiss, W. Troost and T. T. Wu, Nucl. Phys. B264, (1986) 265.

[21] J. Dai, J. F. Gunion and R. Vega, Phys. Lett. B315, (1993) 355; J. Dai, J. F. Gunion and R. Vega, Phys. Lett. B345, (1995) 29; J. Dai, J. F. Gunion and R. Vega, Phys. Lett. B387, (1996) 801.

[22] Data Phys. Rev. D54, (1996) 1. 